Aim of the study: The majority of patients with chronic myeloid leukaemia (CML) respond to tyrosine kinase inhibitors (TKI), while allogeneic haematopoietic cell transplantation (HCT) is indicated in selected clinical situations. HCT carries the risk of severe complications, while the toxicity profile of dasatinib and nilotinib may lead to adverse reactions affecting the quality of life (QoL). We present the results of observational analysis of CML patients who underwent HCT after exposure to second-generation TKI (TKI2), with respect to their quality of life assessed comparatively after transplantation. Material and methods: Eligible subjects included 19 patients. The quality of life and global health assessment were performed with a questionnaire comparing the signs and symptoms present during the TKI2-therapy with those related to post-transplant complications, including psychosocial problems.

Results and conclusions: Most patients had no/few problems with exhausting activities, no/few difficulties during long-distance walks, and do not/rarely rest in the daytime. Seventeen $(89.5 \%)$ patients reported at least one symptom related to TKI2-therapy and most of them disappeared after HCT. Thirteen $(68.4 \%)$ patients noted no serious complication after HCT. Most patients claimed to have a very good QoL and general health compared to the period prior to HCT. We found statistically significant improvement in global health $(p=0.016)$ and QoL $(p=0.043)$ after HCT.

From the survivors perspective, HCT influence positively general health and QoL comparing to TKI2-therapy period. Further studies on larger group of patients will more precisely define the QoL level and possible predictors of changes in QoL, to assess which group of patients needs psychological support.

Key words: tyrosine kinase inhibitors, allogeneic hematopoietic cell transplantation, quality of life.

Contemp Oncol (Pozn) 2016; 20: 414-417 DOI: $10.5114 /$ wo. 2016.64607

\section{Quality of life in chronic myeloid leukaemia patients after haematopoietic cell transplantation pretreated with second-generation tyrosine kinase inhibitors}

Agnieszka Piekarska ${ }^{1}$, Lidia Gil2 ${ }^{2}$ Karolina Jakitowicz ${ }^{1}$, Witold Prejzner ${ }^{1}$, Mieczysław Komarnicki ${ }^{2}$, Andrzej Hellmann ${ }^{1}$

'Department of Hematology and Transplantology, Medical University of Gdańsk, University Clinical Center, Gdansk, Poland

2Department of Haematology and Bone Marrow Transplantation, Poznan University of Medical Sciences, Poznan, Poland

\section{Introduction}

Prior to the era of tyrosine kinase inhibitors (TKI), allogeneic haematopoietic cell transplantation (HCT) was the standard curative therapy for patients with chronic phase $(\mathrm{CP})$ chronic myeloid leukaemia $(\mathrm{CML})$. For more than a decade, the majority of $C M L$ patients have responded to oral targeted therapy, while HCT is indicated in well-defined clinical situations: advanced phase at diagnosis (accelerated phase - AcP; blast phase/crisis - BP) or resistance to imatinib or second-generation TKI (TKI2), e.g. dasatinib, nilotinib [1]. An HCT procedure, giving the hope for long-lasting remission, carries the risk of even fatal complications, while the toxicity profile of both dasatinib and nilotinib may lead to various adverse reactions, affecting the quality of life $(\mathrm{Q} O \mathrm{~L})$ [2-4]. Moreover, prolonged therapy with TKI2 may influence post-transplant complications e.g. development of hepatic veno-occlusive disease (VOD), cardiac toxicity, immune dysfunctions, delayed engraftment, or graft versus host disease (GvHD). The long-term outcome of TKI2-resistant patients remains the issue of their concerns. Potential selection of more aggressive clones may increase the risk of post-HCT relapse and requirement for additional post-transplant therapy [5-8]. Other problems that patients face are caused by the late toxicity of HCT and GVHD-related complications, significantly affecting their quality of life [9].

In this study we present the results of observational analysis of CML patients who underwent HCT after exposure to TKI2, as the second-line therapy, with respect to their quality of life assessed comparatively after transplantation.

\section{Material and methods}

Patients

Eligible subjects were patients with CML, who underwent HCT after pre-treatment with imatinib mesylate and one or more TKI2 in two transplant centres, between February 2008 and November 2013. The therapy with TKI2 was discontinued at least two days prior to the conditioning regimen.

The study was approved by the Bioethical Committee of the Medical University of Gdansk and was conducted in accordance with the Declaration of Helsinki. 


\section{Definitions}

Chronic, accelerated, and blast phases of CML were diagnosed according to ELN criteria [1]. Molecular monitoring was performed according to the ELN guidelines with the RQ-PCR method [10-12]. Deep molecular response $M R^{4.5}$ was defined as a 4.5-log reduction from the baseline ( $\leq 0.0032 \%$ BCR-ABL [IS]) or a disease undetectable in $C D N A$ with the amount of the transcript $A B L \geq 32.000$. Deep $M R^{4.0}$ was defined as a 4-log reduction from the baseline $(\leq 0.01 \%$ BCR-ABL [IS]) or a disease that was undetectable in CDNA with the amount of the transcript $A B L \geq 10.000$. Major molecular response (MMR) was determined when there was a 3 -log reduction from the baseline $(\leq 0.1 \%$ BCR$A B L[I S])$.

Progression/relapse of CML after HCT was diagnosed with the hematologic, cytogenetic or molecular evidence of the disease that required any additional CML-related therapy after HCT (e.g. TKI re-administration and/or donor lymphocyte infusion [DLI]).

Acute graft versus host disease (GvHD) was diagnosed according to IBMTR criteria $[13,14]$. The diagnosis of chronic GvHD was based on NIH consensus criteria [15].

Hepatic VOD was diagnosed with modified Seattle criteria [16].

\section{Quality of life assessment}

The quality of life and subjective global health assessment was performed with the use of a questionnaire based on the standard quality of life scale [17]. Patients were reviewed not earlier than 100 days after HCT. The questionnaire was composed to compare the signs and symptoms present during the TKI2 therapy with those related to post-transplant complications. Patients filled in the questionnaire during their routine control visits in the transplant out-patient.

The first five questions concentrated on everyday activities (carrying things, long and short walks, time spent in bed in daytime, help required in self-service). Patients could choose and sign on the scale one of four possible answers.

The next part was in the form of a table with listed complaints and side effects (e.g. short breath, pain, insomnia, fatigue, weakness, lack of appetite, nausea, vomiting, diarrhoea, worries and fear, sadness, and limits in the everyday and social life) experienced during the TKI2 therapy and subsequently (after HCT). The possible answers were "yes" or "no" for every complaint.

The last part was the patient's estimation (in the form of a scale grading from 1 to 8 ) of their subjective global health and QoL during TKI2 therapy and subsequently.

\section{Statistical analysis}

Differences in mean values between group comparisons were analysed with the Mann-Whitney $U$ test. Data obtained from the questionnaires were presented comparatively with the use of descriptive statistics. Differences in the assessment of global health and QoL were calculated with Pearson chi-square test. $P$-values $<0.05$ were consid- ered statistically significant. Calculations were performed using Stata 13.1 statistical software (StataCorp, TX, USA).

\section{Results}

We prospectively analysed the data of 19 patients treated with the second-generation TKI (dasatinib, nilotinib, or both in sequence) before HCT, who survived at least 100 days after transplantation. Their characteristics are presented in Table 1. The last update of collected data was done in February 2014.

\section{Response to treatment and post-transplant complications}

Seventeen patients achieved $M R^{4.5}$ or $M R^{4.0}$ at a median time of 1.8 months after HCT, and this response was maintained in 13 patients. Two patients achieved MMR, as the best molecular response, at a median time of 1.1 months after HCT. Relapse incidence after HCT in the reviewed group was $21 \%$. Altogether, one haematological relapse and three molecular relapses were diagnosed. Three

Table 1. Patient characteristics

\begin{tabular}{|c|c|}
\hline $\begin{array}{l}\text { Sex, no. (\%) } \\
\text { Male } \\
\text { Female }\end{array}$ & $\begin{array}{l}9(48) \\
10(52)\end{array}$ \\
\hline Median age at HCT (range) & $51(29-68)$ \\
\hline Median age at QoL assessment (range) & $53(31-70)$ \\
\hline $\begin{array}{l}\text { Second-generation TKI (TKI2), no. (\%) } \\
\text { Dasatinib } \\
\text { Nilotinib } \\
\text { Both }\end{array}$ & $\begin{array}{l}9(48) \\
5(26) \\
5(26)\end{array}$ \\
\hline $\begin{array}{l}\text { Disease phase at diagnosis, no. (\%) } \\
\text { Chronic phase } \\
\text { Accelerated phase } \\
\text { Blast phase (crisis) }\end{array}$ & $\begin{array}{l}16(85) \\
2(10) \\
1(5)\end{array}$ \\
\hline $\begin{array}{l}\text { Disease phase at TKI2 treatment, no. (\%) } \\
\text { Chronic phase } \\
\text { Accelerated phase } \\
\text { Blast phase (crisis) }\end{array}$ & $\begin{array}{l}16(85) \\
2(10) \\
1(5)\end{array}$ \\
\hline $\begin{array}{l}\text { Disease phase prior to HCT, no. (\%) } \\
\text { Chronic phase (CP) } \\
\text { Accelerated phase or higher CP } \\
\text { Blast phase (crisis) }\end{array}$ & $\begin{array}{l}13(68) \\
6(32) \\
0(0)\end{array}$ \\
\hline $\begin{array}{l}\text { TKI2 therapy duration prior to HCT, no. (\%) } \\
\text { Median (range) } \\
>12 \text { months } \\
\leq 12 \text { months }\end{array}$ & $\begin{array}{l}18(2-60) \\
12(63) \\
7(37)\end{array}$ \\
\hline $\begin{array}{l}\text { Donor type, no. (\%) } \\
\text { HLA-identical sibling } \\
\text { Matched unrelated }\end{array}$ & $\begin{array}{l}6(32) \\
13(68)\end{array}$ \\
\hline $\begin{array}{l}\text { Conditioning regimen, no. (\%) } \\
\text { MAC (BuCy; TBICy) } \\
\text { RIC (FluMel; FluBu; MelTreoFlu) }\end{array}$ & $\begin{array}{l}7(37) \\
12(63)\end{array}$ \\
\hline $\begin{array}{l}\text { Graft type, no. (\%) } \\
\text { PBSC } \\
\text { BM }\end{array}$ & $\begin{array}{l}16(84) \\
3(16)\end{array}$ \\
\hline
\end{tabular}

HCT - haematopoietic cell transplantation; TK12 - second-generation tyrosine kinase inhibitors; MAC - myeloablative conditioning; $R I C$ - reduced-intensity conditioning; PBSC - peripheral blood stem cells; BM - bone marrow 
Table 2. General health assessment

$\begin{array}{lcc}\begin{array}{l}\text { Score on the scale } \\ (1-8)\end{array} & \begin{array}{c}\text { During TKI2 } \\ \text { therapy no. }(\%)\end{array} & \text { After HCT no. (\%) } \\ 1-2 \text { score } & 0(0) & 0(0) \\ 3-4 \text { score } & 6(31.6) & 1(5.2) \\ 5-6 \text { score } & 11(57.9) & 9(47.4) \\ 7-8 \text { score } & 2(10.5) & 9(47.4)\end{array}$

patients received DLI due to molecular relapses and one due to haematological relapse (combined treatment with $\mathrm{DLI}$ and dasatinib). All of them remain in deep MR until the time of writing.

Mild or moderate VOD was diagnosed in three (16\%) patients from the analysed group, with complete recovery. Acute GvHD (grade 1-2) was observed in two (10\%) patients. In all reviewed patients the timed elapsed from HCT and the presented symptoms enabled us to assess the chronic GVHD incidence and severity. It was diagnosed in nine (47\%) cases, including one person with cGvHD promoted by DLI. Five patients were assessed as mild cGvHD and three patients as moderate cGvHD.

\section{Quality of life}

\section{Assessment of current situation}

Sixteen (84.2\%) patients had no or few problems with exhausting everyday activities. Only three of them experience moderate problems. Fifteen (78.9\%) patients have no or few difficulties during long-distance walks, while only four of them claimed to have moderate difficulties. Shorts walks caused no or just a few problems in all of the questioned subjects. Sixteen (84.2\%) patients did not or rarely needed to rest in bed during the daytime. Three of them sometimes needed to rest in bed during the day. None of the questioned needed help when eating, dressing, washing, or using the toilet.

\section{Assessment of TKI2 toxicity and HCT-related complications}

Seventeen (89.5\%) patients reported at least one symptom related to TKI2 therapy. Most of the symptoms present during TKI2 therapy disappeared after HCT including weakness, lack of appetite, nausea, vomiting, pain, worries, fear, and weakness.

Thirteen (68.4\%) patients noted no serious complication after HCT. Two patients reported occurrence of diarrhoea (10.5\%), one reported weakness (5.2\%), and two reported a little pain (10.5\%) and/or problems with sleeping (10.5\%). Two patients experience some limits in everyday and social life at the time of writing.

\section{Assessment of general health and QoL}

Most patients claimed to have a very good QoL and general health compared to the time prior to HCT. The detailed results of the questionnaire are presented in Table 2 and Table 3. The differences in the assessment of global health
Table 3. Quality of life assessment

$\begin{array}{lcc}\begin{array}{l}\text { Score on the scale } \\ (1-8)\end{array} & \begin{array}{c}\text { During TKI2 } \\ \text { therapy no. }(\%)\end{array} & \text { After HCT no. (\%) } \\ \text { 1-2 score } & 0(0) & 0(0) \\ \text { 3-4 score } & 3(15.9) & 2(10.5) \\ \text { 5-6 score } & 14(73.7) & 8(42.1) \\ 7-8 \text { score } & 2(10.5) & 9(47.4)\end{array}$

$(p=0.016)$ and quality of life $(p=0.043)$ during TKI2 therapy and after HCT are both statistically significant. Moreover, 15 (78.9\%) patients noted at least one grade improvement in their general health and QoL and only two (10.5\%) patients reported one grade worsening in their global health and QoL after HCT.

\section{Discussion}

Therapy with tyrosine kinase inhibitors has been a standard care for patients with CML for more than a decade. Since the majority of patients respond to the therapy and achieve long-lasting deep MR, the group of patients allotransplanted with CML diagnosis is currently limited. In our study we analysed the survivors of HCT procedure, with the follow-up long enough to assess the influence of the most important complications of HCT on QoL. Due to this "preselection", the results concerning the incidence of hepatic VOD and GVHD should not be compared to the whole population of CML patients who undergo HCT. All these data are presented to create the clinical background for QoL analysis.

CML patients require continuous treatment, necessary to control the malignant disease but also related to many adverse reactions that were listed as possible complaints in the questionnaire. The most common adverse reactions of nilotinib include headache, weakness, skin changes, pruritus, nausea, vomiting, diarrhoea, symptoms secondary to cytopaenias (anaemia, thrombocytopaenia, neutropaenia), abnormalities in electrocardiography (ECG), increase of liver enzymes, and lipase in serum. A nilotinib daily dose ranges from $600 \mathrm{mg}$ to $800 \mathrm{mg}$ given twice daily on an empty stomach one hour before the meal or two hours after the meal. The list of most serious adverse reactions of dasatinib includes drug-related fluid retention (superficial oedema, pleural effusion), headache, weakness, skin changes, nausea, vomiting, diarrhoea, consequences of cytopaenia, and muscular and joint pain. A dasatinib daily dose ranges from $50 \mathrm{mg}$ to $140 \mathrm{mg}$ with no meal-associated scheduling. As dasatinib and nilotinib tablets contain lactose, patients with lactose intolerance may experience adverse symptoms from the GI tract. Patients are aware that they must tolerate all of the "inconveniences" of TKI2 therapy for many years, probably until the end of their life, and this fact can be frustrating for some of them. Furthermore, there is no guarantee of long-lasting remission, especially in the case of more advanced phase of the disease at diagnosis. 
The patients reviewed in our study faced and survived a life-threatening procedure of allogeneic haematopoietic cell transplantation that must have had a certain impact on their physical and psychological condition. After HCT, they also experience the fear of a possible relapse and consequences of HCT-related complications, e.g. acute and chronic GvHD or recurrent infections. On the other hand, they know that immunosuppressive and anti-infectious therapy is used temporally and most patients do not require any treatment one year after HCT. The limited time of post-transplant treatment increases the patients' sense of "being healthy" or even cured from leukaemia. That is why the questionnaire was created to assess the current physical condition, and then it enabled a comparison with certain problems before HCT (during TKI2 therapy) and after HCT (subsequently). The results of the study showed that HCT procedure, from the survivors' perspective, positively influenced not only their general health, but also their evaluation of QoL.

We conclude that, in the view of the curative potential of HCT procedure and non-inferior QoL, HCT is the optimal option for CML patients with defined indications. In contrast to adverse reactions of TKI2-therapy, transplant-related complications are usually not permanent, and, therefore, acceptable for the patients. The presented results show some directions in the level of QoL but, nonetheless, further observation of more numerous samples is required to assess the individual influence of nilotinib and dasatinib on the transplant-related toxicity, HCT outcome, and QoL. Moreover, studies on larger groups of patients will more precisely define the level of QoL, as well as possible predictors of changes in QoL, to identify those who need psychological support.

The authors declare no conflict of interest.

\section{References}

1. Baccarani M, Deininger MW, Rosti G, et al. European LeukemiaNet recommendations for the management of chronic myeloid leukemia: 2013. Blood 2013; 122: 872-84.

2. Garg RJ, Kantarjian H, O’Brien S, et al. The use of nilotinib or dasatinib after failure to 2 prior tyrosine kinase inhibitors: long-term follow-up. Blood 2009; 114: 4361-8.

3. Kim TD, le Coutre P, Schwarz M, et al. Clinical cardiac safety profile of nilotinib. Haematologica 2012; 97: 883-9.

4. Valent P. Severe adverse events associated with the use of second-line $B C R / A B L$ tyrosine kinase inhibitors: preferential occurrence in patients with comorbidities. Haematologica 2011; 96: 1395-7.

5. Deol A, Lum LG. Role of donor lymphocyte infusions in relapsed hematological malignancies after stem cell transplantation revisited. Cancer Treat Rev 2010; 36: 528-38.

6. Saussele S, Lauseker M, Gratwohl A, et al. Allogeneic hematopoietic stem cell transplantation (allo SCT) for chronic myeloid leukemia in the imatinib era: evaluation of its impact within a subgroup of the randomized German CML Study IV. Blood 2010; 115: 1880-5.

7. Shanavas M, Messner HA, Kamel-Reid S, et al. A comparison of long-term outcomes of donor lymphocyte infusions and tyrosine kinase inhibitors in patients with relapsed CML after allogeneic hematopoietic cell transplantation. Clin Lymphoma Myeloma Leuk 2014; 14: 87-92.
8. Wadhwa J, Szydlo RM, Apperley JF, et al. Factors affecting duration of survival after onset of blastic transformation of chronic myeloid leukemia. Blood 2002; 99: 2304-9.

9. Eapen M, Logan BR, Appelbaum FR, et al. Long-term survival after transplantation of unrelated donor peripheral blood or bone marrow hematopoietic cells for hematologic malignancy. Biol Blood Marrow Transplant 2015; 21: 55-9.

10. Beillard E, Pallisgaard N, van der Velden VH, et al. Evaluation of candidate control genes for diagnosis and residual disease detection in leukemic patients using 'real-time' quantitative reverse-transcriptase polymerase chain reaction (RQ-PCR) - a Europe against cancer program. Leukemia 2003; 17: 2474-86.

11. Cross NC, White HE, Müller MC, Saglio G, Hochhaus A. Standardized definitions of molecular response in chronic myeloid leukemia. Leukemia 2012; 26: 2172-5.

12. Gabert J, Beillard E, van der Velden VH, et al. Standardization and quality control studies of real-time' quantitative reverse transcriptase polymerase chain reaction of fusion gene transcripts for residual disease detection in leukemia - a Europe Against Cancer program. Leukemia 2003; 17: 2318-57.

13. Przepiorka D, Weisdorf D, Martin P, Klingemann HG, Beatty P, Hows J, Thomas ED. 1994 Consensus Conference on Acute GVHD Grading. Bone Marrow Transplant 1995; 15: 825-8.

14. Rowlings PA, Przepiorka D, Klein JP, et al. IBMTR Severity Index for grading acute graft-versus-host disease: retrospective comparison with Glucksberg grade. Br J Haematol 1997; 97: 855-64.

15. Filipovich AH, Weisdorf D, Pavletic S, et al. National Institutes of Health consensus development project on criteria for clinical trials in chronic graft-versus-host disease: I. Diagnosis and staging working group report. Biol Blood Marrow Transplant 2005; 11: 945-56.

16. Carreras E. Veno-occlusive disease of the liver after hemopoietic cell transplantation. Eur J Haematol 2000; 64: 281-91.

17. Burckhardt CS, Anderson KL. The Quality of Life Scale (QOLS): reliability, validity, and utilization. Health Qual Life Outcomes 2003; 1: 60

\section{Address for correspondence}

\section{Agnieszka Piekarska}

Department of Hematology and Transplantology

Medical University of Gdańsk, University Clinical Center

Debinki 7

80-952 Gdańsk, Poland

e-mail: babajaga@gumed.edu.pl

Submitted: 21.05 .2015

Accepted: $\quad 9.10 .2015$ 